\title{
Magnetic field amplification by the small-scale dynamo in the early Universe
}

\author{
Jacques M. Wagstaff, ${ }^{1,}$ | Robi Banerjee, ${ }^{1}$ Dominik Schleicher, ${ }^{2}$ and Günter Sigl ${ }^{3}$ \\ ${ }^{1}$ Hamburger Sternwarte, Gojenbergsweg 112, 21029 Hamburg, Germany \\ ${ }^{2}$ Institut für Astrophysik, Georg-August-Universität Göttingen, \\ Friedrich-Hund-Platz 1, 37077 Göttingen, Germany \\ ${ }^{3}$ II Institut für Theoretische Physik, Universität Hamburg, \\ Luruper Chaussee 149, 22761 Hamburg, Germany
}

(Dated: October 29, 2018)

\begin{abstract}
In this paper we show that the Universe is already strongly magnetized at very early epochs during cosmic evolution. Our calculations are based on the efficient amplification of weak magnetic seed fields, which are unavoidably present in the early Universe, by the turbulent small-scale dynamo. We identify two mechanisms for the generation of turbulence in the radiation dominated epoch where velocity fluctuations are produced by the primordial density perturbation and by possible first-order phase transitions at the electroweak or QCD scales. We show that all the necessities for the small-scale dynamo to work are fulfilled. Hence, this mechanism, operating due to primordial density perturbations, guarantees fields with comoving field strength $B_{0} \sim 10^{-6} \varepsilon^{1 / 2} \mathrm{nG}$ on scales up to $\lambda_{c} \sim 0.1 \mathrm{pc}$, where $\varepsilon$ is the saturation efficiency. The amplification of magnetic seed fields could be even larger if there are first-order phase transitions in the early Universe. Where, on scales up to $\lambda_{c} \sim 100 \mathrm{pc}$, the comoving field strength due to this mechanism will be $B_{0} \sim 10^{-3} \varepsilon^{1 / 2} \mathrm{nG}$ at the present time. Such fields, albeit on small scales, can play an important role in structure formation and could provide an explanation to the apparently observed magnetic fields in the voids of the large-scale structure.
\end{abstract}

\section{INTRODUCTION}

Magnetic fields of strengths of order a few $\mu \mathrm{G}$ have been observed in galaxies at high and low redshifts, in galaxy clusters and in superclusters 175 . There is also evidence for strong extragalactic magnetic fields coming from $\gamma$-ray observations. These studies place a lower bound on intergalactic magnetic fields at $3 \times 10^{-7} \mathrm{nG}$ [6], although plasma effects may complicate the propagation of electromagnetic cascades [7, 8,

Theoretically, magnetic fields are very likely to have been generated at some level in the early Universe through a variety of mechanisms. On the largest scales, magnetic fields can be generated during inflation giving today $B_{0} \sim\left(10^{-25}-10^{-1}\right) \mathrm{nG}$ on a scale of $1 \mathrm{Mpc}$ [9]. However, such mechanisms require some modification to the Maxwell theory in order to break its conformal invariance. Without such modifications, fields of present strengths $B_{0} \sim\left(10^{-20}-10^{-11}\right) \mathrm{nG}$ could have been generated at the electroweak (EW) and QCD phase transitions respectively [10]. In this case, the magnetic field coherence length is limited by the particle horizon size at the time of generation, typically much smaller than $1 \mathrm{Mpc}$. At later times, magnetic fields could have been generated through the generation of vorticity in the primordial plasma [11 15] (originally proposed by Harrison [16]). This mechanism is very natural, since vorticity in the plasma is unavoidably generated in the late radiation era through the nonlinear couplings of first-order density perturbations. The seed fields generated here are of order $B_{0} \sim 10^{-20} \mathrm{nG}$.

\footnotetext{
* jwagstaff@hs.uni-hamburg.de
}

In many cases, the observed magnetic fields are much stronger than fields predicted by theories. Therefore, in order to explain observations, some amplification of the generated seed fields must have occurred at some point in the history of the Universe. A popular mechanism for such amplification is known as the dynamo mechanism.

The dynamo mechanism comes in two broad classes (see Ref. 17] for a review). The large-scale dynamo converts kinetic energy on large scales into magnetic energy. This mechanism can act if the conducting fluid flow is highly helical, inhomogeneous or anisotropic, the typical example being the differential rotation of galaxies. This galactic dynamo, which operates only for spiral galaxies, requires seed fields of order $B_{0} \sim 10^{-21} \mathrm{nG}$ to obtain $\mu \mathrm{G}$ strengths today [18. However, this type of dynamo cannot explain strong fields in much younger galaxies, in galaxy clusters and superclusters or indeed in the voids of the large-scale structure.

The second class of dynamo works with stationary, homogeneous and isotropic turbulence. This mechanism, known as the small-scale dynamo (SSD), also converts kinetic energy from turbulent motions into magnetic energy and typically operates on much smaller scales. Magnetic field lines, which are frozen into the conducting plasma, are stretched, twisted and folded by the random motions of fluid elements leading to exponential field amplification. The SSD mechanism has been applied to the formation of the first stars and galaxies in the matter dominated Universe [19, 20, (see Ref. 21] for an early discussion on this subject), where the turbulent motions arise from gravitational collapse, accretion and supernovae explosions (e.g. Refs. [20, 22, 25]). If turbulence is predominantly injected by supernova explosions, this mechanism may further explain the observed corre- 
lation between the star formation rate and the magnetic field strength in spiral galaxies 26. This mechanism can be highly effective at magnetizing structures in the early Universe. However, a problem evades explanation; the large field strengths apparently observed in the voids of the large-scale structure [6].

In this paper we investigate the SSD amplification of magnetic seed fields in the radiation dominated Universe. If significant turbulence is generated in this early epoch, then small magnetic seed fields could be amplified very efficiently by the mechanism. Unlike velocity perturbations, magnetic fields survive through the viscous damping and free-streaming regimes. Therefore, the SSD could be an effective mechanism to strongly magnetize the early Universe prior to structure formation, leading to strong intergalactic magnetic fields. We demonstrate that the conditions are right for efficient dynamo amplification leading to large magnetic fields, albeit on very small scales, which could explain observations and have an impact on early structure formation.

The structure of our paper is as follows. In Sec. II we give a brief review of the small-scale dynamo mechanism and the conditions necessary for its action. In Sec. III we look at two mechanisms for the generation of turbulence in the radiation dominated era. In Sec. [V] we investigate the amplification of magnetic fields due to SSD action and look at their subsequent evolution to the present time. We summarize in Sec. V and conclude in Sec. VI.

\section{THE SMALL-SCALE DYNAMO MECHANISM}

The small-scale dynamo (SSD) mechanism is a very efficient mechanism at converting kinetic energy from turbulent motions to magnetic energy [27, 28]. To describe the mechanism, we first briefly review the conditions necessary for turbulence to arise.

The kinetic Reynolds number $R_{e}$ characterizes the relative importance of the fluid advective and dissipative terms in the Euler equation. For random motions correlated on some physical scale $l$ with root-mean-square (rms) velocity $v_{l}^{\text {rms }}$, the local kinetic Reynolds number is given by 29,30 ,

$$
R_{e}(l)=\left\{\begin{array}{cll}
\frac{v_{l}^{\mathrm{rms}} l}{\eta_{s}} & \text { if } \quad l \gg l_{\mathrm{mfp}} \\
\frac{v_{l}^{\mathrm{rms}}}{\alpha_{\mathrm{d}} l} & \text { if } \quad l \ll l_{\mathrm{mfp}}
\end{array}\right.
$$

for dissipation due to diffusing particles and freestreaming particles respectively, where $l_{\mathrm{mfp}}$ is the particle mean-free-path (mfp). Here, $\eta_{s}$ is the shear viscosity and $\alpha_{\mathrm{d}}$ is a drag coefficient due to the occasional scattering of fluid particles [30, 31]. On a given scale $l$, a viscous regime corresponds to $R_{e}(l) \ll 1$. Whereas for a turbulent regime $R_{e}(l) \gg 1$, in this case the dissipative time scale is much greater than the eddy-turnover time scale $\tau_{l}$, where

$$
\tau_{l} \equiv l / v_{l}^{\mathrm{rms}}=a l_{c} / v_{l}^{\mathrm{rms}},
$$

and $l_{c}$ is a comoving length.

With the injection of kinetic energy, a turbulent flow develops almost inevitably when the kinetic Reynolds numbers are large enough [32, 33]. Indeed, there is a critical value for which turbulence is expected, i.e. $R_{e}(l) \gtrsim R_{e}^{\mathrm{cr}} \sim 10^{3}$. The fundamental reasons for the transition to a turbulent flow are not completely understood. However, flow instabilities always arise when the Reynolds numbers are larger than the critical value [32]. One possible mechanism for the triggering of the flow instability is due to thermal fluctuations [34], which are important in the radiation epoch, but other mechanisms may exist too. Indeed, in this paper we will look at turbulence driven by bubble collisions in first-order phase transitions.

Turbulence is characterized by a direct cascade of energy from large scales to small scales. The eddy-turnover time gives the time over which eddy flows break down to smaller scales in this direct cascade. With a continuous injection of kinetic energy (or forcing/stirring of the fluid) at the forcing/stirring scale $L$, turbulence becomes fully developed (or stationary) on a time scale of order the eddy-turnover time scale at the forcing scale $\tau_{L}$. In an expanding Universe, this time scale must be at most given by the Hubble time i.e. $\tau_{L}=1 / H$. Thus, the largest possible forcing scale is $L=v_{L}^{\mathrm{rms}} / H$, where $v_{L}^{\mathrm{rms}}$ is the typical velocity fluctuation on the forcing scale $L$. The velocity spectrum for fully developed turbulence is then given by

$$
v_{l}^{\mathrm{rms}}=v_{L}^{\mathrm{rms}}\left(\frac{l}{L}\right)^{\vartheta}
$$

The scaling index $\vartheta$ varies between the two extremes $1 / 3 \leq \vartheta \leq 1 / 2$, where for incompressible Kolmogorov type turbulence $\vartheta=1 / 3$ and for highly compressible Burgers type turbulence $\vartheta=1 / 2$. This spectrum is valid only on the inertial range: $l_{\text {diss }}<l<L$, which is determined by the turbulent kinetic energy cascade. The dissipative scale $l_{\text {diss }}$ is the scale at which turbulent velocities are diffused due to viscosity at the same rate as they are replenished from larger scales. At this scale the direct cascade ends. The dissipative scale can be defined through $R_{e}\left(l_{\text {diss }}\right) \sim 1$, hence $l_{\text {diss }} \sim L R_{e}(L)^{-1 /(\vartheta+1)}$ assuming $l_{\text {diss }} \gg l_{\mathrm{mfp}}$ in Eq. (1).

The SSD mechanism converts this turbulent kinetic energy to magnetic energy [27, 28]. The effectiveness of the mechanism depends strongly on three important environmental factors (i) the Reynolds number; stronger turbulence is more effective (ii) the turbulent velocity modes; rotational modes are much more efficient than longitudinal modes [35, 36] and (iii) the Prandtl number $P_{m} \equiv R_{m} / R_{e}=4 \pi \sigma \eta_{s}$, where the Prandtl number is a measure of the relative importance of the magnetic and kinetic diffusion. Here, the $R_{m}$ is the magnetic Reynolds 
number given by

$$
R_{m}(l)=4 \pi \sigma a l_{c} v_{l}^{\mathrm{rms}},
$$

and $\sigma$ is the plasma conductivity.

There are two competing effects in the turbulent dynamo mechanism; magnetic field line stretching and resistive reconnection. The critical magnetic Reynolds number $R_{m}^{\text {cr }}$ defines the balance between the stretching and reconnection. For $R_{m}(L)<R_{m}^{\text {cr }}$ reconnection wins and there is no dynamo and for $R_{m}(L)>R_{m}^{\text {cr }}$ the stretching wins and the dynamo takes effect amplifying the magnetic field. Independent of the Prandtl number, the critical magnetic Reynolds number is $R_{m}^{\mathrm{cr}} \approx 60$ and $R_{m}^{\text {cr }} \approx 2700$ for Kolmogorov and Burgers type turbulence respectively [17, 36, 37. When the SSD takes effect, the fluctuating component of the magnetic field grows exponentially $B_{\mathrm{rms}} \propto \exp (\Gamma t)$ due to turbulence in a weakly magnetized plasma. Depending on the Prandtl number, the growth rate $\Gamma$ scales with either the kinetic Reynolds number $R_{e}$ or the magnetic Reynolds number $R_{m}$, in particular $\Gamma \propto R_{e}^{(1-\vartheta) /(1+\vartheta)}$ and $\Gamma \propto R_{m}^{(1-\vartheta) /(1+\vartheta)}$ for $P_{m} \gg 1$ and $P_{m} \ll 1$ respectively [36, 37.

There is a large number of numerical studies in the literature that have demonstrated the SSD action unambiguously for a number of settings [35, 38, 41]. Analytically, the Kazantsev model (following the formalism by Refs. [17, 42]) was developed in order to study the evolution of magnetic fields in a conducting plasma containing turbulent motions [28. The Kazantsev model can be used to calculate the magnetic field growth rate and the critical magnetic Reynolds number required for SSD action. The model considers random turbulent motions correlated on a scale $l$ with velocity $v_{l}^{\text {rms }}$ and spectrum given in Eq. (3) valid on the inertial range $l_{\text {diss }}<l<L$. The spectrum of velocity fluctuations is assumed to be Gaussian, homogeneous and isotropic in space and instantaneously correlated in time. The kinetic Reynolds number must also be larger than some critical value for which turbulence is expected, i.e. $R_{e}(L) \gtrsim R_{e}^{\mathrm{cr}} \sim 10^{3}$ (this is a conservative estimate for $R_{e}^{\mathrm{cr}}$, indeed the SSD action has been observed in cases where $R_{e}(L) \lesssim 100[38,40]$ ). By modeling the turbulent velocity spectrum so that it behaves as Kolmogorov or Burgers turbulence for scaling index $\vartheta=1 / 3,1 / 2$ respectively, it can be shown that the magnetic field growth rate is given by [36, 43 .

$$
\Gamma=\frac{(163-304 \vartheta)}{60} R_{e}(L)^{(1-\vartheta) /(1+\vartheta)} \tau_{L}^{-1} .
$$

This result is valid in the large Prandtl number limit $P_{m} \gg 1$, which is relevant to cosmological plasmas. Since the Reynolds numbers are typically very large, the magnetic fields can be amplified very rapidly. We also note that this analytical result for the growth rate has been verified by Ref. 43] via a numerical integration of the Kazantsev equation.

The phase of rapid exponential amplification comes to an end when the magnetic energy becomes comparable to the kinetic energy on the dissipative scale $l_{\text {diss }}$. The system then enters a stage of nonlinear growth, where the magnetic field grows as some power law in time $B_{\mathrm{rms}}(t) \propto t^{\vartheta /(1-\vartheta)}$ 44. This phase lasts until the magnetic field is saturated on the forcing scale $L$. Saturation is given by the approximate equipartition between magnetic and kinetic energy $E_{\mathrm{M}} / E_{\text {kin }} \approx \varepsilon$. This occurs when

$$
\left\langle\boldsymbol{B}^{2}(\boldsymbol{x})\right\rangle \approx 4 \pi \varepsilon(\rho+p)\left\langle\boldsymbol{v}^{2}(\boldsymbol{x})\right\rangle,
$$

where the parameter $\varepsilon$ quantifies the saturation efficiency. Numerical studies (for $P_{m} \approx 2$ ) indicate that the SSD mechanism is more efficient for rotational modes, where the saturation efficiency $\varepsilon$ is close to unity [35]. Whereas the saturation level is lower for compressive modes $\varepsilon \sim 10^{-3}-10^{-4}$ [35]. However, further numerical work is required to establish the saturation level for large Prandtl numbers. So far there are no analytical results to determine the efficiency parameter $\varepsilon$.

Here, we stress that the SSD mechanism is a rather generic phenomenon, in the sense that the mechanism works independently of the type of turbulence [36, 45, 46]. In particular, it is interesting to note that even purely irrotational turbulence can still drive a small-scale dynamo. This was originally shown by Ref. [46] and later Ref. [36] reached similar conclusions. Hence, the efficient amplification of magnetic fields seems unavoidable if any kind of turbulence is generated in a magnetized plasma.

\section{TURBULENCE IN THE EARLY UNIVERSE}

In this section we use Eq. (1) to calculate the Reynolds numbers in the radiation dominated (RD) era in order to identify epochs of turbulence. With large Reynolds numbers, as we have argued in the previous section that any injection of kinetic energy into the plasma will lead to a state of fully developed turbulence for a range of scales. In the RD era, the kinetic Reynolds number in the diffusive regime is given by

$$
R_{e}(l, T)=\frac{5 g_{*}(T)}{g_{\nu, \gamma}} \frac{v_{l}^{\mathrm{rms}} l_{c}}{l_{\mathrm{mfp}, c}^{\nu, \gamma}(T)},
$$

where the shear viscosity $\eta_{s}=\left(g_{\nu, \gamma} / 5 g_{*}\right) l_{\mathrm{mfp}}^{\nu, \gamma}$ is determined by the particles of longest mean free path $l_{\mathrm{mfp}}^{\nu, \gamma}$, which are either neutrinos or photons depending on the time. Here, $g_{*}$ and $g_{\nu, \gamma}$ are the total and component number of effective relativistic degrees of freedom.

In the very early Universe, before neutrino decoupling $T \gtrsim 2.6 \mathrm{MeV}$, neutrinos have the longest mfp and are thus most efficient at transporting momentum and heat. At high temperatures the shear viscosity due to neutrinos is low and the plasma could be in a turbulent regime $R_{e} \gg 1$. At this time, the comoving mfp is 31]

$$
l_{\mathrm{mfp}, c}^{\nu} \simeq \frac{a^{-1}}{G_{F}^{2} T^{2}\left(n_{l}+n_{q}\right)},
$$


which is proportional to $1 / T^{4}$. Here $n_{l}=6 g_{l} \zeta(3) T^{3} / 7 \pi^{2}$ and $n_{q}=6 g_{q} \zeta(3) T^{3} / 7 \pi^{2}$ are the lepton and quark number densities, $g_{l, q}$ are the number of degrees of freedom for relativistic leptons and quarks, $\zeta$ is the Riemann zeta function and $G_{F}$ is the Fermi constant. However, the neutrino mfp increases as the Universe expands and cools, leading to a viscous regime $R_{e}<1$ (see e.g. Ref. 30). Eventually the neutrinos decouple at $T_{\mathrm{dec}} \simeq 2.6 \mathrm{MeV}$. From here on, momentum and heat is effectively transported by the photons. At early times, photons generate a small shear viscosity in the plasma and the fluid flow could become turbulent once again. The comoving photon mfp is given by [47.

$$
l_{\mathrm{mfp}, c}^{\gamma} \simeq \frac{a^{-1}}{\sigma_{T}\left(n_{\mathrm{pair}}^{2}+n_{e}^{2}\right)^{1 / 2}}
$$

where $\sigma_{T}=8 \pi \alpha^{2} / 3 m_{e}^{2}$ is the Thomson cross section, $\alpha \approx 1 / 137$ is the fine structure constant and $m_{e}$ is the electron mass. During this epoch, the number densities $n_{\text {pair }}$ and $n_{e}$ of $e^{ \pm}$pairs and free electrons respectively are given by [47]

$$
\begin{aligned}
n_{\text {pair }} & \approx\left(\frac{2 m_{e} T}{\pi}\right)^{3 / 2} \exp \left(-\frac{m_{e}}{T}\right)\left(1+\frac{15}{8} \frac{T}{m_{e}}\right), \\
n_{e} & =X_{e} \frac{\Omega_{b} \rho_{0}}{m_{p}}\left(\frac{T}{T_{0}}\right)^{3}
\end{aligned}
$$

where $m_{p}$ is the proton mass, the baryon fraction and present day density product is $\Omega_{b} \rho_{0} \simeq 1.81 \times 10^{-12} \mathrm{eV}^{4}$ [48, $T_{0} \simeq 2.725 \mathrm{~K}$ is the present day photon temperature and the ionization fraction is $X_{e}=1$ in the RD era.

Once the temperature decreases below the electron mass $T<m_{e} \simeq 0.511 \mathrm{MeV}, e^{ \pm}$pairs begin to annihilate and the photon mfp increases rapidly. The $e^{ \pm}$annihilation completes at around $T \simeq 20 \mathrm{keV}$. After this, as the temperature drops further, photons begin to diffuse followed by photon drag and the fluid is in a viscous regime once again 30, 31. Hence, there are two epochs in the $\mathrm{RD}$ era, before and after neutrino decoupling, where the Reynolds numbers could be large and turbulence is potentially fully developed for a range of scales.

Diffusing particles also damp away velocity fluctuations (see Sec. IIIC). Therefore, before diffusion sets in, there is always a possibility for the plasma to be in a turbulent state if the considered scales are large enough. However, the eddy-turnover time increases for larger scales. We must therefore look at the evolution of all relevant scales carefully in order to establish whether or not turbulence is possible. To complete the calculation for the Reynolds numbers, we must estimate the turbulent velocity fluctuations in the early Universe. In the next two subsections we present two mechanisms for the generation of turbulence in the $\mathrm{RD}$ era.

\section{A. Turbulence from primordial density perturbations}

To explain the formation of the large-scale structure in the Universe observed today, a primordial density perturbation of magnitude $\delta \rho / \rho \sim 10^{-5}$ is required at the time of matter-radiation equality [29]. The primordial density perturbation is thought to have been generated at a much earlier time and therefore must be present in the very early Universe during the RD era. Cosmic inflation provides the most compelling explanation for the origin of the primordial perturbation [49].

Well before horizon entry, the primordial curvature perturbation, which determines the gravitational potential $\Phi$, remains constant and given by the initial condition $\Phi_{0}$. In the Newtonian gauge, its equation of motion is $\Phi^{\prime \prime}+3 \mathcal{H}(1+w) \Phi^{\prime}-w \nabla^{2} \Phi=0$, where $p=w \rho$, $\mathcal{H}=a^{\prime} / a$ and ${ }^{\prime} \equiv \partial / \partial \eta$ with conformal time $\eta$ [50. In the RD era $w=1 / 3$, the solution for the Fourier modes reads $\Phi(\boldsymbol{k}, \eta)=3\left[j_{1}(y) / y\right] \Phi_{0}(\boldsymbol{k})$, where $y \equiv k \eta / \sqrt{3}, k$ is the comoving wave number and $j_{1}(y)=\sin y / y^{2}-\cos y / y$ is the first spherical Bessel function. As a Fourier mode of the gravitational potential reenters the horizon during the RD era, it begins to oscillate with an amplitude decreasing as $1 / y^{2} \propto 1 / t$.

The initial conditions, which are probed by observations, are given by the two-point correlation function

$$
\left\langle\Phi_{0}\left(\boldsymbol{k}_{1}\right) \Phi_{0}^{*}\left(\boldsymbol{k}_{2}\right)\right\rangle=(2 \pi)^{3} P_{\Phi}(k) \delta^{3}\left(\boldsymbol{k}_{1}-\boldsymbol{k}_{2}\right),
$$

where $P_{\Phi}(k)=\left(2 \pi^{2} / k^{3}\right)(9 / 25) \Delta_{\mathcal{R}}^{2}\left(k_{0}\right)\left(k / k_{0}\right)^{n_{s}-1}$. The results of the Planck mission give $\Delta_{\mathcal{R}}^{2}\left(k_{0}\right)=2.215 \times 10^{-9}$ and $n_{s} \simeq 0.96$ for the pivot scale $k_{0}=0.05 \mathrm{Mpc}^{-1}$ [48.

Perturbations in the fluid 3-velocity field are generated by the density perturbations. At first-order in density perturbations, the fluid velocity perturbation is purely irrotational (curl free) with Fourier modes 50.

$$
\begin{aligned}
v_{i}(\boldsymbol{k}, \eta) & =-\frac{i k_{i}}{2 \mathcal{H}^{2}}\left[\Phi^{\prime}(\boldsymbol{k}, \eta)+\mathcal{H} \Phi(\boldsymbol{k}, \eta)\right] \\
& =-i \frac{3 \sqrt{3}}{2} \hat{k}_{i}\left[\sin y-2 j_{1}(y)\right] \Phi_{0}(k) .
\end{aligned}
$$

These modes $v_{i}$ oscillate with the density perturbation, but have a term which does not decay with the expansion.

Let us define the spectrum of velocity perturbations in Fourier space by the two-point correlation function

$$
\left\langle v_{i}\left(\boldsymbol{k}_{1}, \eta\right) v_{i}^{*}\left(\boldsymbol{k}_{2}, \eta\right)\right\rangle=(2 \pi)^{3} \frac{2 \pi^{2}}{k^{3}} \mathcal{P}_{v}(k) \delta^{3}\left(\boldsymbol{k}_{1}-\boldsymbol{k}_{2}\right) .
$$

Hence, with Eq. 14 and the spectrum $P_{\Phi}$ we find

$$
\mathcal{P}_{v}(k)=\frac{243}{100}\left[\sin y-2 j_{1}(y)\right]^{2} \Delta_{\mathcal{R}}^{2}\left(k_{0}\right)\left(k / k_{0}\right)^{n_{s}-1} .
$$

The velocity spectrum oscillates rapidly for subhorizon scales $y, k / \mathcal{H} \gg 1$, therefore we can average $\mathcal{P}_{v}(k)$ over many oscillations. For a scale invariant primordial spectrum $n_{s}=1$ we find $\overline{\mathcal{P}}_{v}(k) \simeq \frac{243}{200} \Delta_{\mathcal{R}}^{2}\left(k_{0}\right)$. Hence, on 
subhorizon scales, the spectrum of velocity perturbations generated by first-order density perturbations is isotropic, homogeneous, Gaussian and to a good approximation scale invariant, see Fig. 1 .

The root-mean-square ( $\mathrm{rms}$ ) velocity is then given by

$$
v^{\mathrm{rms}} \equiv \sqrt{\left\langle\boldsymbol{v}^{2}(\boldsymbol{x})\right\rangle}=\left(\int_{0}^{\infty} \mathcal{P}_{v}(k) \frac{d k}{k}\right)^{1 / 2} .
$$

It will also be useful to define the rms velocity on a given comoving length scale $l_{c}=2 \pi / k_{l}$ by

$$
v_{l}^{\mathrm{rms}} \simeq\left(\int_{k_{l}}^{k_{\mathrm{diss}}} \mathcal{P}_{v}(k) \frac{d k}{k}\right)^{1 / 2}
$$

where $l_{\text {diss }, c}=2 \pi / k_{\text {diss }}$ is a cut-off or dissipative scale. The typical value of the velocity perturbation is therefore

$$
v_{l}^{\mathrm{rms}} \sim \sqrt{\overline{\mathcal{P}}_{v}(k)} \simeq \sqrt{\Delta_{\mathcal{R}}^{2}\left(k_{0}\right)} \simeq 5 \times 10^{-5}
$$

where natural units are used such that $c=1$.

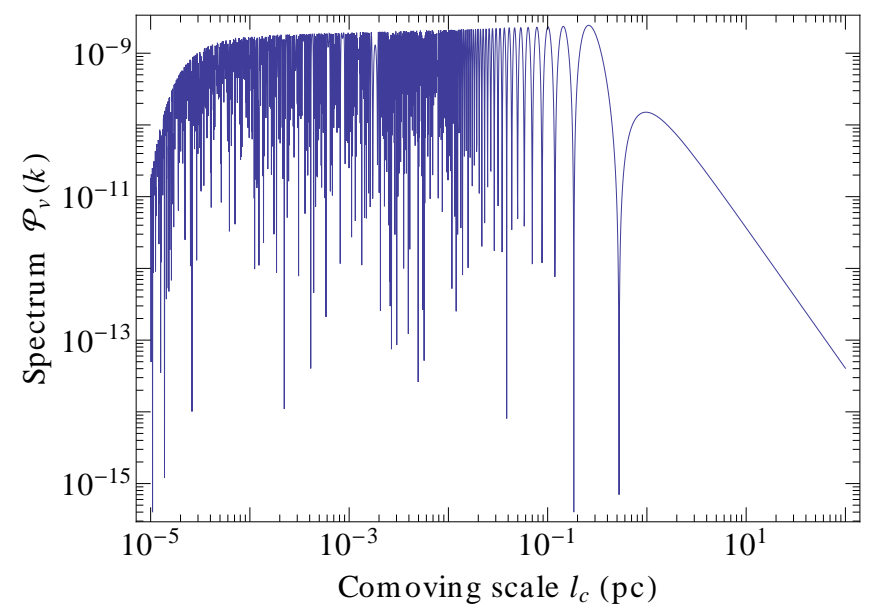

FIG. 1. The spectrum of velocity perturbations, given in Eq. (16), at a temperature $T \simeq 0.21 \mathrm{GeV}$. The figure shows how the velocity perturbations are generated upon horizon entry (at around $l_{c} \simeq 0.31 \mathrm{pc}$ ) and are scale invariant until damping due to neutrino diffusion takes over at around $l_{c} \simeq 1.5 \times 10^{-5} \mathrm{pc}$ (see Sec. III C) .

In the radiation dominated era, new $k$-modes of the primordial density perturbation, which generate velocity perturbations, are continuously reentering the horizon. This continuous production of velocity perturbations can be seen as the continuous forcing of the fluid on the largest scales. Thereby, if the Reynolds numbers are large enough, turbulent flow will occur. The velocity perturbations forcing the fluid are purely longitudinal in this case. However, rotational modes will be generated at second order in cosmological perturbations [51 55]. In any case, with $R_{e} \gg 1$, nonlinear interactions can play a role in generating turbulence with both longitudinal and rotational fluid motions. Therefore, a state of fully developed (or stationary) turbulence can be expected on a time scale of order the eddy-turnover time scale at the forcing scale $\tau_{L}$ i.e. $\tau_{L}=1 / H$ in an expanding Universe (see Sec. II). Hence, for all scales below $L=v_{L}^{\mathrm{rms}} / H$, there are many eddy-turnover times per Hubble time $\tau_{l} \lesssim 1 / H$. This condition ensures interactions between eddy flows leading to fully developed turbulence with a spectrum given in Eq. (3). A second condition is that the Reynolds number on the forcing scale $L$ is larger than some critical value for which turbulence is expected, i.e. $R_{e}(L) \gtrsim R_{e}^{\mathrm{cr}} \sim 10^{3}$. With these two conditions we find the range of scales, corresponding to the inertial range, $l_{\text {diss }} \lesssim l \lesssim L$, where $R_{e}\left(l_{\text {diss }}\right) \sim 1$. In Sec. IIID we establish when, in the RD era, and on what scales, the Reynolds numbers are large given the turbulent velocity fluctuations generated by the primordial density perturbations.

\section{B. Turbulence injected from phase transitions}

In this section we briefly describe another mechanism for the generation of turbulence in the RD era. The mechanism occurs during first-order phase transitions when bubbles of the new phase collide and merge 56 58. In the early Universe, the electroweak and QCD phase transitions are potentially first order, although under early Universe conditions with very small chemical potentials the QCD transition is a smooth transition whereas the electroweak transition could be first order in certain Standard Model extensions. These violent phenomena can inject large kinetic energy into the plasma, thereby generating turbulence and allowing the possibility of small-scale dynamo action.

The characteristic time scale for the phase transition is given by the rate of bubble nucleation $\beta^{-1}$. Here $\beta$ is expected to be $\beta \sim 100 H$ [58]. The largest bubbles reach a size $\beta^{-1} v_{b}$ by the end of the phase transition, where $v_{b}$ is the bubble wall expansion velocity. Thus, we take $L \simeq \beta^{-1} v_{b}$ as the largest stirring scale and $\tau_{\text {stir }}=\beta^{-1}$ as the stirring time scale 58 . The phase boundary can propagate via two modes, deflagration and detonation, where the wall velocity $v_{b}$ is subsonic and supersonic respectively [56, 57]. It has been argued in the literature that deflagrations are unstable to becoming detonations via bubble wall instabilities [59. Hence, for simplicity we will only consider detonations, where the wall velocity is fully determined and given by 56 .

$$
v_{b}(\alpha)=\frac{1}{1+\alpha}\left(\frac{1}{\sqrt{3}}+\sqrt{\alpha^{2}+\frac{2 \alpha}{3}}\right) .
$$

Here $\alpha \equiv \rho_{\text {vac }} / \rho_{\text {thermal }}$ determines the strength of the phase transition. In this case, the fraction of vacuum energy converted to kinetic energy $\kappa \equiv \rho_{\text {kin }} / \rho_{\text {vac }}$ takes the form [57]

$$
\kappa(\alpha)=\frac{1}{1+0.72 \alpha}\left(0.72 \alpha+\frac{4}{27} \sqrt{\frac{3 \alpha}{2}}\right) .
$$


For phase transitions that give large stirring times compared to the eddy turnover time of the largest scale $\tau_{\text {stir }} \gg \tau_{L}$, a direct cascade of energy is set up and a state of fully developed turbulence is established in a time scale $\tau_{L}$ and can be expected for a duration time $\tau_{\text {stir }}$ [58. Since the rate of energy dissipation is equal to the mean input power in stationary turbulence, the amplitude of the Kolmogorov spectrum can be easily determined. This calculation is done in Ref. [58, where they show that $v_{L}^{\mathrm{rms}} \simeq\left(\alpha \kappa v_{b}\right)^{1 / 3}$ and argue that $v_{L}^{\mathrm{rms}} \lesssim 1 / \sqrt{3}$. Hence, the condition for this simpler case $\tau_{\text {stir }} \gg \tau_{L}$ is translated to $3 v_{b} \ll \sqrt{2 \alpha \kappa}$ [58, 60] which is satisfied only for $\alpha \gtrsim 1$. Therefore, in this case of strong detonation $\alpha \gtrsim 1$ we have $v_{L}^{\mathrm{rms}} \sim 1$ [57]. However, if $\tau_{\text {stir }} \lesssim \tau_{L}$, a state of turbulence can still be expected [58]. The stirring corresponds to an impulsive force acting on the plasma that will cascade down to smaller scales. Eddy flows on large scales $L$ act as a source for eddies on smaller scales for a duration time $\tau_{L}$. Following Ref. [60, in the time scale $\tau_{L}$, we neglect the decay of the turbulence and assume a state of fully developed turbulence for a duration time $\tau_{L}$ [58. Numerical work in Ref. 61] has established that kinetic energy in the form of acoustic waves persist well beyond the time of the phase transition. The nonlinear interaction of these acoustic waves could also be a source of turbulence on larger time scales. In the weak detonation limit $\alpha \lesssim 1$, we find [57, 58.

$$
v_{L}^{\mathrm{rms}} \simeq \frac{\sqrt{2 \alpha \kappa}}{3(2 \pi)^{4 / 3}} .
$$

Hence, for first-order phase transitions of strengths in the range $\alpha \sim\left(10^{-5}-10^{-1}\right)$, we find turbulent velocities

$$
v_{L}^{\mathrm{rms}} \sim\left(10^{-4}-10^{-1}\right) .
$$

In this case, the turbulence is expected to be of Kolmogorov type.

\section{Damping of turbulence}

Velocity perturbations of the baryonic fluid are damped below a scale $l_{D}$ due to particles diffusing out of overdense regions. The damping is very efficient and given by (see for example Ref. 62] )

$$
v \propto \exp \left[-\left(\frac{l_{D}^{\nu, \gamma}}{l_{c}}\right)^{2}\right],
$$

where $l_{c}$ is a comoving length scale. In the $\mathrm{RD}$ era, the comoving damping scale due to neutrinos or photons random walking out of perturbations is given by 29,62 .

$$
\left(l_{D}^{\nu, \gamma}\right)^{2} \simeq \int_{0}^{t} \frac{l_{\mathrm{mfp}, c}^{\nu, \gamma}\left(t^{\prime}\right)}{a\left(t^{\prime}\right)} \mathrm{d} t^{\prime},
$$

where $l_{\mathrm{mfp}, c}^{\nu, \gamma}$ is the comoving particle mean-free-path $(\mathrm{mfp})$. The efficient damping of velocity perturbations is seen in the spectrum in Fig. 1. This important effect must be considered carefully when we come to investigate the scales of turbulence in the RD era (see next section). Here, we briefly note that although turbulent velocity fluctuations are efficiently damped due to diffusing particles, magnetic fields become overdamped and survive through such viscous and free-streaming regimes (this effect is discussed in detail in Sec. IV B) [30, 31, 63.

\section{The scales of turbulence}

The evolution of relevant scales from the time of the electroweak (EW) scale $T_{\mathrm{EW}} \sim 100 \mathrm{GeV}$ to the time of neutrino decoupling $T_{\mathrm{dec}} \simeq 2.6 \mathrm{MeV}$ is shown in Fig. 2. In this early epoch the neutrinos generate the plasma viscosity. The QCD phase transition occurs at around $T_{\mathrm{QCD}} \simeq 200 \mathrm{MeV}$. The scales of interest are the comoving Hubble scale $l_{H}=1 / a H$, the largest stirring scale $L_{c}=v_{L}^{\mathrm{rms}} / a H$ with the values $v_{L}^{\mathrm{rms}}$ from primordial density perturbations (PDP) and first-order phase transitions (PT) in eqs. 19) and 23) respectively, and the damping scale due to neutrino diffusion $l_{D}^{\nu}$ [cf. Eq. (25)]. Here, we assume that the damping scale due to neutrino diffusion is the only relevant damping scale at this time i.e. the velocity perturbations on small scales are not damped due to physical processes at higher temperatures. Indeed, this is a safe assumption since neutrinos are the most weakly interacting particles in the Standard Model and at early times are the most efficient heat transporters.

Let us first consider the turbulence generated by the primordial density perturbations (PDP). From Fig. 2 we can see that for $T \gtrsim 0.2 \mathrm{GeV}$ the stirring scale $L_{c, \text { PDP }}$ (the lower blue dotted line in the figure) is larger than the damping scale $l_{D}^{\nu}$. Hence, the velocity perturbations are not damped and we can use the value given in Eq. (19). With this value for the $v_{L}^{\mathrm{rms}}$ we can calculate the Reynolds numbers $R_{e}\left(L_{c}\right)$ from Eq. (7), these are shown in Fig. 3 (the lower dotted blue line). We find that $R_{e}\left(L_{c}\right) \gg 1$ for $0.2 \lesssim T / \mathrm{GeV} \lesssim 100$. The largest stirring scale, over which large Reynolds numbers are found, is roughly given at $T \simeq 0.2 \mathrm{GeV}$ i.e. $L_{c, \mathrm{PDP}} \sim 10^{-5} \mathrm{pc}$. Hence, at these times, between the damping scale $l_{D}^{\nu}$ and $L_{c, \text { PDP }}$, we expect a state of fully developed turbulence. However, for $T \lesssim 0.2 \mathrm{GeV}$, the scale $L_{c \text {,PDP }}$ is below the damping scale $l_{D}^{\nu}$, which means that velocity perturbations generated by the primordial density perturbation are exponentially damped [cf. Eq. (24)] and so are the Reynolds numbers. Thus, below this temperature, the plasma is in a viscous regime and there is no turbulence.

For turbulence generated by first-order phase transitions (PT), the stirring scale can be much larger (the upper dotdashed blue line in Fig. 2), since the velocity fluctuations can be much stronger [cf. Eq. 230]. Hence, if the phase transition occurs at any time in the epoch between $T \sim\left(10^{2}-10^{-3}\right) \mathrm{GeV}$, a state of turbulence can be expected. In Fig. 3, the Reynolds numbers are shown 


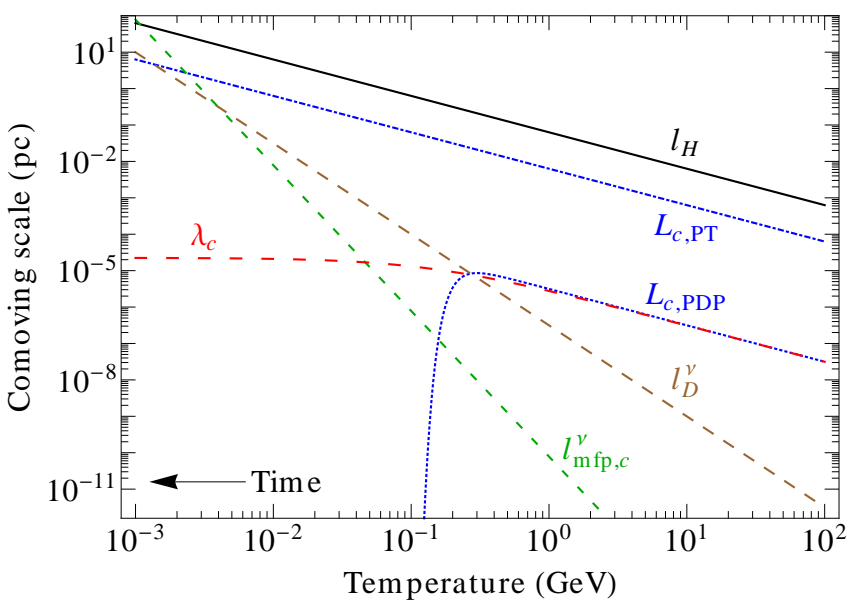

FIG. 2. This figure shows the evolution of relevant comoving scales from the EW scale $T_{\mathrm{EW}} \sim 100 \mathrm{GeV}$ to the time of neutrino decoupling at $T_{\mathrm{dec}} \simeq 2.6 \mathrm{MeV}$. In this early epoch the neutrinos generate the plasma viscosity. The QCD phase transition occurs at around $T_{\mathrm{QCD}} \simeq 200 \mathrm{MeV}$. Here, $l_{H}=1 / a H$ is the Hubble scale (solid, black), $l_{\mathrm{mfp}, c}^{\nu}$ is the neutrino mean-free-path (dashed, green) and $l_{D}^{\nu}$ is the damping scale due to neutrino diffusion given by Eq. 25) (dashed, brown). For turbulence generated by the primordial density perturbation (PDP) and first-order phase transitions (PT), the largest stirring scales $L_{c}=v_{L}^{\mathrm{rms}} / a H$ are shown with the values $v_{L}^{\text {rms }}$ from eqs. (19) and (23) (dotted, blue) and (dotdashed, blue) respectively. In Eq. (23), the upper value for $v_{L}^{\mathrm{rms}}$ is used. Although the turbulent motions from PDP become completely damped below $T \simeq 0.2 \mathrm{GeV}$, the magnetic field gets frozen-in with integral scale $\lambda_{c}$ (dashed, red).

to be very large between the EW and QCD scales, indicating a highly turbulent state. The largest stirring scale, over which large Reynolds numbers are found, is roughly given by the horizon size at that time of the phase transition: $1 /\left.a H\right|_{\mathrm{QCD}} \sim 0.1 \mathrm{pc}$ and $1 /\left.a H\right|_{\mathrm{EW}} \sim 10^{-4} \mathrm{pc}$ for the QCD and EW phase transitions respectively.

The evolution of relevant scales from the time of neutrino decoupling $T_{\mathrm{dec}} \simeq 2.6 \mathrm{MeV}$ to a time long after $e^{ \pm}$ annihilation $T \simeq 100 \mathrm{eV}$ is shown in Fig. 4. In this epoch the photons generate the plasma viscosity. The scales of interest are the comoving Hubble scale $l_{H}=1 / a H$, the largest stirring scale $L_{c, \mathrm{PDP}}=v_{L}^{\mathrm{rms}} / a H$ with the value $v_{L}^{\text {rms }}$ from Eq. 19) and both damping scales $l_{D}^{\nu, \gamma}$ given by Eq. (25). The largest damping scale due to neutrino diffusion (which occurs at an earlier time) is approximately given by the particle horizon at the time of neutrino decoupling i.e. $l_{D}^{\nu} \approx 1 /\left.a H\right|_{\mathrm{dec}} \simeq 42 \mathrm{pc}$ 31. Here, we assume that, if there is indeed a first-order phase transition, it would have occurred at a much earlier time. Therefore, in this epoch, turbulence can only be generated by the primordial density perturbations.

However, with the value of the rms velocity from Eq. 19) damped only by photon diffusion, we can clearly see from Fig. 4 that the scale $L_{c, \text { PDP }}$ is below the largest damping scale due to neutrino diffusion $l_{D}^{\nu} \approx 42 \mathrm{pc}$ throughout this epoch. Therefore, the velocity pertur-

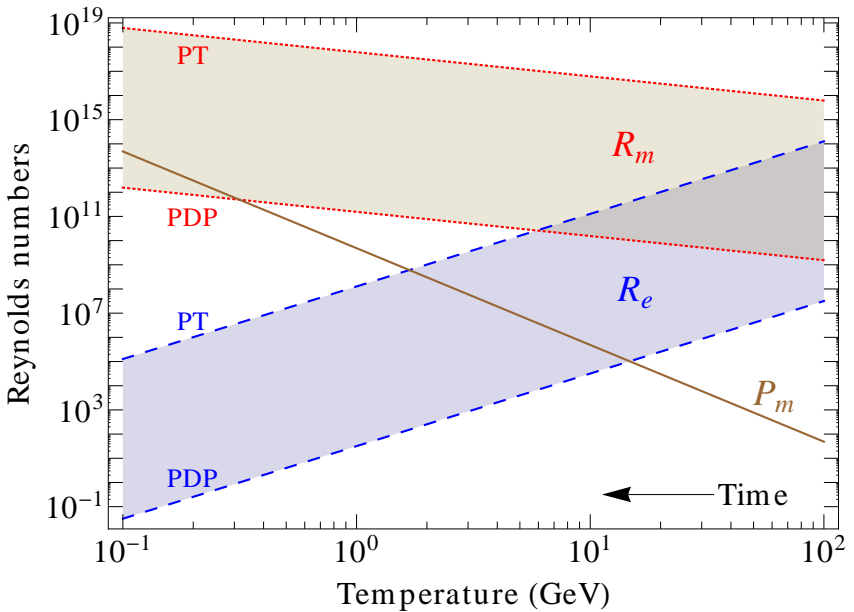

FIG. 3. This figure shows the evolution of the different Reynolds numbers from the EW scale $T_{\mathrm{EW}} \sim 100 \mathrm{GeV}$ to the QCD scale $T_{\mathrm{QCD}} \simeq 200 \mathrm{MeV}$. The kinetic Reynolds number $R_{e}\left(L_{c}\right)$ (dashed, blue) is determined from eqs. (7) and (8) and the magnetic Reynolds number $R_{m}\left(L_{c}\right)$ (dotted, red) from eqs. (4) and (26). The lower lines for the Reynolds numbers correspond to turbulence generated by the primordial density perturbations (PDP) i.e. using the (undamped) value for $v_{L}^{\mathrm{rms}}$ from Eq. (19). The upper lines for the Reynolds numbers correspond to turbulence generated by first-order phase transitions (PT) with the (undamped) value $v_{L}^{\mathrm{rms}} \sim 10^{-1}$ from Eq. (23). This figure clearly shows that $R_{e} \gg 1$ in all cases, suggesting that the plasma is in a state of fully developed turbulence during this time. The figure also shows the Prandtl numbers $P_{m}=R_{m} / R_{e} \gg 1$ (solid, brown).

bations are efficiently damped by particle diffusion [cf. Eq. (24)]. Indeed, on the scale $L_{c, \text { PDP }}$, the velocity perturbations generated by the primordial density perturbations are completely erased due to neutrino diffusion at this time. Hence, the kinetic Reynolds numbers become vanishingly small even when the shear viscosity is very small. Thus, at these temperatures, the plasma is in a viscous regime and there is no turbulence.

\section{EVOLUTION OF COSMOLOGICAL MAGNETIC FIELDS}

In this section we consider the cosmological evolution of magnetic fields from the time of their generation to the present day (see for example Refs. [30, 31, 63. ). We first consider the amplification of magnetic seed fields due to small-scale dynamo (SSD) action in the early Universe. Then, we consider the subsequent evolution to the present time. In order to compare with observations, it is important to theoretically determine the final magnetic field strength and coherence length. 


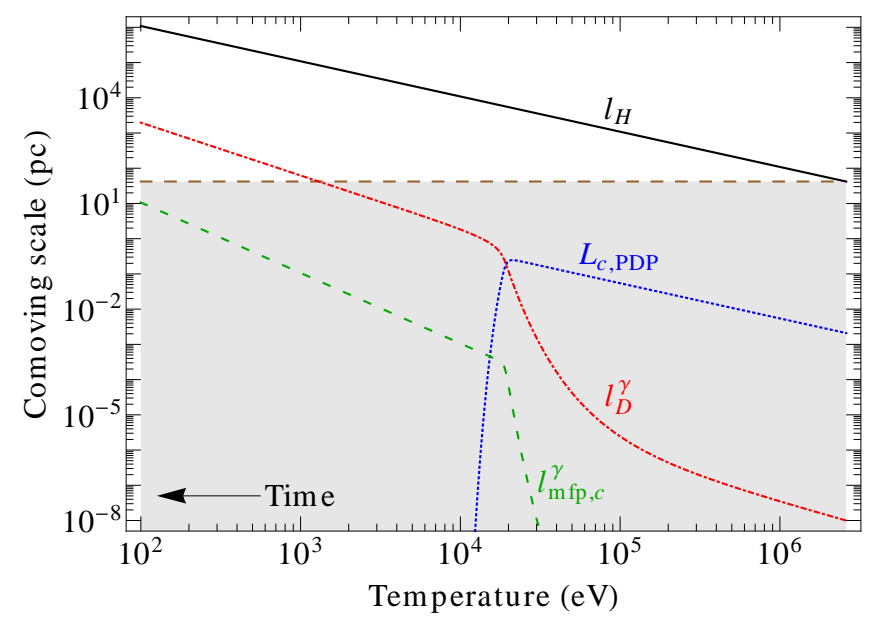

FIG. 4. This figure shows the evolution of relevant comoving scales from the time of neutrino decoupling at $T_{\text {dec }} \simeq 2.6 \mathrm{MeV}$ to a time long after $e^{ \pm}$annihilation at $T \simeq 100 \mathrm{eV}$. In this epoch the photons generate the plasma viscosity. The time of $e^{ \pm}$annihilation occurs at $m_{e} \gtrsim T \gtrsim 20 \mathrm{keV}$. Here, $l_{H}=1 / a H$ is the Hubble scale (solid, black), $l_{\mathrm{mfp}, c}^{\gamma}$ is the photon meanfree-path (dashed, green), $L_{c, \mathrm{PDP}}=v_{L}^{\mathrm{rms}} / a H$ (dotted, blue) with the value $v_{L}^{\mathrm{rms}}$ from Eq. $(19)$, and $l_{D}^{\gamma}$ is the damping scale due to photons (dotdashed, red) given by Eq. 25). Velocity fluctuations below the scale $l_{D}^{\nu} \approx 1 /\left.a H\right|_{\text {dec }} \simeq 42 \mathrm{pc}$ (shaded area) are damped due to neutrino diffusion at an earlier time, see Fig. 2 Hence, below $l_{c} \approx 42 \mathrm{pc}$, the velocity perturbations generated by the primordial density perturbations are completely erased and no turbulence can be generated in this epoch.

\section{A. Amplification by small-scale dynamo action}

In Sec. III we identified two mechanisms that generate turbulence in the early Universe. In a turbulent and weakly magnetized plasma, small magnetic seed fields can be amplified exponentially through the SSD action (as described in Sec. II). Let us now assume that small magnetic seed fields exist at the time of the EW phase transition. These fields may have been generated at the phase transition [10] or at an earlier time (for example during inflation [9]). We now investigate the possibility of SSD action in the RD era.

The injection of kinetic energy, together with large Reynolds numbers, leads to a state of fully developed turbulence. In the previous section we found that turbulence is expected due to primordial density perturbations or first-order phase transitions at high temperatures, between $0.2 \lesssim T / \mathrm{GeV} \lesssim 100$ (see Fig. 3). Indeed, we expect fully developed turbulence below the stirring scale $L_{c}=v_{L}^{\mathrm{rms}} / a H$. At these temperatures, the conductivity $\sigma$ is given by 64]

$$
0.76 T \lesssim \sigma \lesssim 6.7 T
$$

where the larger value corresponds to the upper temperature bound. With the above we can calculate the Prandtl numbers and the magnetic Reynolds numbers. These are also shown in Fig. 3. The Prandtl numbers at these times are very large, $P_{m} \sim\left(10^{2}-10^{12}\right)$, which means that we can neglect dissipative effects due to finite conductivity throughout the epoch of interest. From Eq. (4), we find the magnetic Reynolds numbers $R_{m}\left(L_{c}\right) \sim\left(10^{9}-10^{12}\right)$ for turbulence generated by primordial density perturbations and a maximum range of $R_{m}\left(L_{c}\right) \sim\left(10^{16}-10^{18}\right)$, using the upper value $v_{L}^{\text {rms }} \sim 0.1$ in Eq. (23), for turbulence generated by first-order phase transitions. The large magnetic Reynolds numbers, $R_{m} \gg R_{m}^{\text {cr }} \approx 60$ (for Kolmogorov turbulence) [17, indicate that we are well within the regime where the SSD mechanism is expected to operate.

Figure 5 shows the magnetic field growth rate $\Gamma$, where $B_{\mathrm{rms}} \propto \exp (\Gamma t)$, which is determined from the Kazantsev model of the SSD mechanism and given in Eq. (5). The growth rate depends on the type of turbulence, where $\vartheta=1 / 3,1 / 2$, applicable on the inertial range $l_{\text {diss }}<l<L$ [cf. Eq. (33]], for Kolmogorov and Burgers type turbulence respectively. Here, we assume that the turbulence is of Kolmogorov type, which is relevant for the subsonic velocity fluctuations determined in this paper. Now, since $\Gamma$ varies in time, it will be useful to consider the number of $e$-foldings given by $N \equiv \int \Gamma(t) \mathrm{d} t$. In Fig. 5, the growth rate is shown in units of the turnover rate of the largest eddy $\tau_{L}^{-1}$ and $N(T)$ is shown where $\Gamma(T)$ is integrated from $T=100 \mathrm{GeV}$ to temperature $T$. Since the magnetic field grows as $B_{\mathrm{rms}} \propto \exp (\Gamma t)$, the number of $e$-foldings gives the total amplification factor. Due to very large Reynolds numbers, the growth rate is initially very large and the number of $e$-foldings quickly becomes large. Hence, we find a very rapid increase in the field strength and a huge amplification factor leading to rapid saturation.

The phase of rapid exponential amplification comes to an end when the magnetic energy becomes comparable to the kinetic energy. In the radiation dominated epoch, this saturation occurs when

$$
\left\langle\boldsymbol{B}^{2}(\boldsymbol{x})\right\rangle \approx \frac{8}{45} \pi^{3} \varepsilon g_{*} T^{4}\left\langle\boldsymbol{v}^{2}(\boldsymbol{x})\right\rangle,
$$

where $g_{*}$ is the total number of effective relativistic degrees of freedom, and the parameter $\varepsilon$ quantifies the saturation efficiency (see Sec. III). Without further dynamical evolution, the field strength will only be diluted by the expansion $B \propto a^{-2} \propto T^{2}$. Let us assume that the magnetic field becomes saturated on the largest forcing scale $L_{c}$. Thus, redshifted to present day values we find

$$
B_{0}^{\mathrm{rms}}=a^{2} B_{\mathrm{rms}} \approx \sqrt{\frac{8}{45} \pi^{3} \varepsilon g_{*}\left(T_{*}\right)} T_{0}^{2} v_{L}^{\mathrm{rms}}
$$

where $T_{0}$ is the present day photon temperature and $T_{*}$ is the radiation temperature at the time of magnetic field amplification. With this simple assumption, we find that in order to saturate magnetic seed fields of strength $B_{0}^{\text {seed }} \simeq\left(10^{-30}-10^{-20}\right) \mathrm{nG}$ up to $\mathcal{O}(1)$ nG level we require $e$-folding numbers $N \simeq 46-70$. 


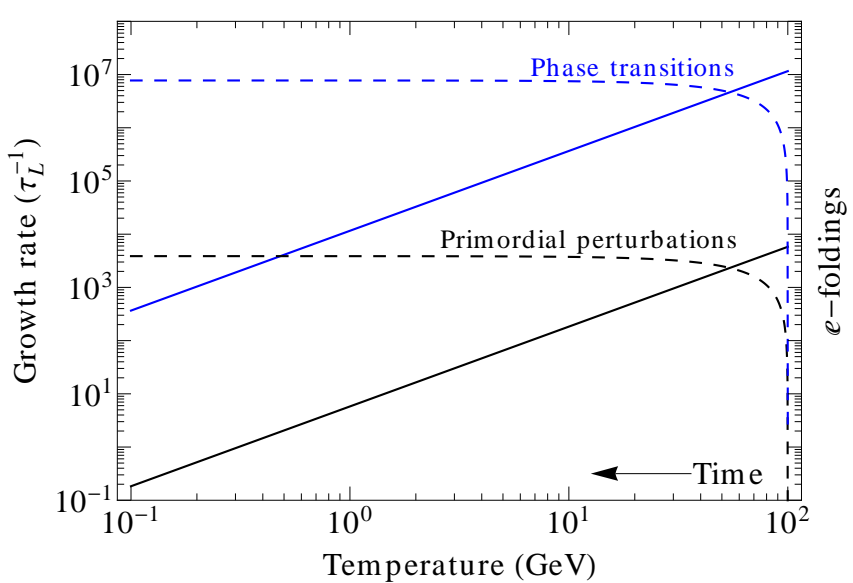

FIG. 5. This figure shows the SSD growth rate $\Gamma$ (solid) and number of $e$-foldings $N=\int \Gamma(t) d t$ (dashed) from the time of the EW scale $T_{\mathrm{EW}} \sim 100 \mathrm{GeV}$ to the QCD scale $T_{\mathrm{QCD}} \simeq 200 \mathrm{MeV}$. Recall that the magnetic seed field is amplified as $B_{\text {rms }} \propto \exp (\Gamma t)$. The growth rate, shown here in units of $\tau_{L}^{-1}$, is determined from Eq. (5) where Kolmogorov turbulence $\vartheta=1 / 3$ is assumed. The growth rate and the number of $e$-foldings for turbulence generated by the primordial density perturbation and first-order phase transitions are shown in (black) and (blue) respectively.

We can now estimate the saturated field strength from Eq. (28). For turbulence generated by the primordial density perturbation, the kinetic energy is given by the typical velocity fluctuations in Eq. (19). Hence, we find a saturated magnetic field strength whose value today is $B_{0}^{\mathrm{rms}} \approx 1 \times 10^{-9} \varepsilon^{1 / 2} \quad$ G. For turbulence generated by first-order phase transitions, the kinetic energy is given by the velocity fluctuations in Eq. (23) i.e. $v_{L}^{\mathrm{rms}} \sim\left(10^{-4}-10^{-1}\right)$. Hence, we find the saturated field strengths in the range $B_{0}^{\mathrm{rms}} \approx\left(10^{-3}-1\right) \varepsilon^{1 / 2} \mu \mathrm{G}$.

Here we note that the amplification up to saturation of magnetic seed fields from kinetic energy injected at firstorder phase transitions has been considered previously in the literature, see for example Ref. [10] and references within. However, we believe it is important to point out that there is a well-defined and clearly described dynamo process that does the amplification, namely the smallscale dynamo theory provides the relevant framework for predictions regarding growth rates and saturation levels.

In the case where turbulence is generated by the primordial density perturbation, the fluid forcing is continuous and turbulence is fully developed throughout this epoch. Hence, as we can see from Fig. 5, the number of $e$-foldings quickly become very large $N \gtrsim \mathcal{O}(100)$ and there is enough time in this epoch for tiny magnetic seed fields to saturate. However, for turbulence generated by first-order phase transitions, the fluid forcing is not continuous. In Sec. III A we argue that for $\alpha \lesssim 1$, the time scale for the duration of turbulence is approximately $\tau_{L}$ [58. Therefore, the number of $e$-foldings $N \equiv \int \Gamma d t$ is roughly given by $\Gamma / \tau_{L}^{-1} \approx R_{e}^{1 / 2} \gg 1$ (see Eq. (5) assuming a Kolmogorov spectrum). Hence, the magnetic fields can easily become saturated in the time scale of the phase transition.

The saturated field strengths are very strong. However, the scales over which we expect saturation are very small. We expect saturation on scales from the damping scale $l_{D}$ up to the largest forcing scale $L_{c}$. For a Kolmogorov spectrum, where most power resides on the largest scale, we can identify $L_{c}$ as the comoving coherence length $\lambda_{c}$ of the magnetic field. For turbulence generated by the primordial density perturbation, the largest forcing scale in the epoch considered is $L_{c}$ at $T \simeq 0.2 \mathrm{GeV}$ i.e. $\quad \lambda_{c} \sim 10^{-5} \mathrm{pc}$ (see Fig. 2). For turbulence generated by first-order phase transitions, the length scales on which saturation is expected depends on the exact time of the phase transition. The basic constraint on the coherence length is the horizon size at the time of the phase transition; $\lambda_{c} \simeq 0.1 \mathrm{pc}$ and $\lambda_{c} \simeq 10^{-4} \mathrm{pc}$ for the QCD and EW phase transitions respectively.

To obtain larger coherence lengths, the magnetic field would need to be amplified and saturated at a later time when the Hubble horizon is larger and turbulence develops on larger scales. Unfortunately, the velocity perturbations generated by the primordial density perturbation are efficiently damped below $T \simeq 0.2 \mathrm{GeV}$ and therefore do not lead to a state of fully developed turbulence. Without turbulence there is no SSD action and therefore no amplification of primordial magnetic fields. For the SSD mechanism to be effective at a later time in the RD era, a different mechanism which injects kinetic energy into the plasma is required.

\section{B. Subsequent evolution}

In the previous section we considered the amplification of magnetic seed fields at the time when turbulent kinetic energy is injected into the primordial plasma. The integral scale of the magnetic field, which is amplified up to equipartition with the kinetic energy, is determined by the scale of the injected turbulence. In this section we consider the cosmological evolution of the magnetic field strengths and coherence lengths from the time after the injection of turbulence to the present time. We follow the works of Refs. 30, 31, 63, 65, 66 for the growth of the coherence length and the damping of turbulence in this subsequent regime in order to determine the final magnetic field strengths and coherence lengths. The most important result from such works is that magnetic fields generated in early epochs survive through viscous and free-streaming regimes, unlike velocity perturbations which are efficiently damped (see Sec. IIIC).

In the turbulent regimes, strong magnetic fields on small scales drive turbulence in the plasma up to equipartition. The turbulence removes power on small scales thereby increasing the correlation length and reducing the field strength. This turbulent magnetohydrodynamic (MHD) effect, free turbulent decay, depends on the type of turbulence generated and on whether or not the mag- 
netic field is helical [30, 63. For nonhelical fields, the growth of the coherence length is purely due to the dissipation of power on small scales. In the helical case, there is an inverse cascade effect where the power on larger scales grows [30, 67.

Let us only consider nonhelical magnetic fields in turbulent regimes. The growth of $\lambda_{c}$ is a power law in time with an index that depends on the magnetic field spectrum $n_{B}$. For magnetic fields generated by causal processes, e.g. phase transitions, the index is $n_{B}=2$ [68]. In this case $\lambda_{c} \sim t^{2 / 7}, t^{2 / 5}$ and the magnetic field strength at the scale $\lambda_{c}$ evolves as $a^{2} B_{\text {rms }} \sim t^{-5 / 7}, t^{-3 / 5}$ for incompressible (Kolmogorov) and compressible (Burgers) type turbulence respectively [30, 63, 65, 66]. Hence, the evolution of the field strength and coherence length up to the time of recombination is determined by the relation

$$
a^{2} B_{\mathrm{rms}} \sim \lambda_{c}^{-n}
$$

where $n=\frac{5}{2}, \frac{3}{2}$ for Kolmogorov and Burgers turbulence respectively [30, 63, 65, 66].

Besides the evolution of magnetic fields in the turbulent regimes, in the $\mathrm{RD}$ era, there are also epochs of viscous damping and free-streaming. In a magnetized plasma, there are different modes in which magnetic energy can be stored; fast, slow and Alfvén modes, as opposed to only the acoustic mode in the case of velocity fluctuations. The fast magnetosonic mode decays in the same manner as the acoustic mode due to particle diffusion, see Eq. 25. However, the slow and Alfvén modes evolve differently and can become overdamped 31. Hence, the magnetic energy stored in these modes becomes frozen-in. The overdamping depends on the scales and magnetic field strength. Therefore, magnetic fields survive through viscous and free-streaming regimes, which is in contrast to turbulent velocity fluctuations that become efficiently damped. In the viscous damping and free-streaming regimes, the evolution of $a^{2} B_{\text {rms }}$ and $\lambda_{c}$ is halted until free turbulent decay begins again [30, 63]. The evolution due to free turbulent decay terminates when the correlation length and field strength end on the line, in the $\left\{a^{2} B_{\mathrm{rms}}, \lambda_{c}\right\}$ plane, given by 30,63 .

$$
a^{2} B_{\mathrm{rms}} \simeq 10^{-8} \frac{\lambda_{c}}{\mathrm{Mpc}} \mathrm{G} .
$$

This line corresponds to the largest eddies being processed at recombination $1 /\left(a_{\text {rec }} H_{\text {rec }}\right) \simeq \lambda_{c} / v_{A}$ with $v_{A}$ the Alfvén speed 63. In the matter dominated Universe, there is no further evolution of the magnetic field correlation length $\lambda_{c}$, although strong fields on small scales can drive turbulence at much later times in the intergalactic medium and restore the turbulent decay [63. In this sense, Eq. (30) becomes an upper bound on the present day magnetic field strength. The difference in evolution of the magnetic modes, in contrast to acoustic modes, means that fields generated and amplified in the radiation dominated era can survive to the present day.
As seen in the previous subsection, turbulence generated by the primordial density perturbation can amplify tiny magnetic seed fields through the SSD mechanism to values of order $a^{2} B_{\text {rms }} \sim 1 \varepsilon^{1 / 2} \mathrm{nG}$ on scales at most $\lambda_{c} \sim 10^{-5}$ pc. From eqs. 29 and 30, these fields would evolve to $a^{2} B_{\mathrm{rms}} \sim 10^{-6} \varepsilon^{1 / 2} \mathrm{nG}$ on scales $\lambda_{c} \sim 10^{-1}$ pc. Since the primordial density perturbation is necessarily present for structure formation, such fields are guaranteed by the SSD mechanism and can play an important role in structure formation. Unfortunately, these fields are too weak on too short scales to explain the Fermi observations of $\mathrm{TeV}$ Blazars [6]. However, turbulence generated by first-order phase transitions can amplify magnetic seed fields to values of order $a^{2} B_{\text {rms }} \sim\left(10^{-3}-1\right) \varepsilon^{1 / 2} \quad \mu \mathrm{G}$ on scales $\lambda_{c} \sim\left(10^{-4}-10^{-1}\right) \mathrm{pc}$. These initial field configurations will evolve to $a^{2} B_{\text {rms }} \sim\left(10^{-6}-10^{-3}\right) \varepsilon^{1 / 2} \mathrm{nG}$ on scales $\lambda_{c} \sim\left(10^{-1}-10^{2}\right)$ pc. Such fields are strong enough to explain the Fermi observations of TeV Blazars [6].

\section{SUMMARY}

In this paper we have identified two mechanisms that generate turbulence in the radiation dominated Universe. The two mechanisms inject kinetic energy into the primordial plasma at times when the kinetic Reynolds numbers are very large $R_{e} \gg 1$. With the injection of kinetic energy, which is determined by $v_{L}^{\mathrm{rms}}$, and large Reynolds numbers, a state a fully developed turbulence is expected on the inertial range: $l_{\text {diss }, c}<l_{c}<L_{c}$, where $L_{c}=v_{L}^{\mathrm{rms}} / a H$ is the largest forcing scale and $l_{\text {diss }, c} \sim L_{c} R_{e}(L)^{-3 / 4}$ is the dissipative scale (for Kolmogorov turbulence). The two mechanisms for generating velocity fluctuations are: by the primordial density perturbation and bubble collisions during first-order phase transitions. In Sec. IV A, we have seen that turbulence is inevitably generated by the primordial density perturbation prior to the QCD scale $T \gtrsim 200 \mathrm{MeV}$. In Sec. IIIB, we investigated the possibility of turbulence generated at first-order phase transitions between the electroweak (EW) and the QCD scales $200 \mathrm{MeV} \lesssim T \lesssim 100 \mathrm{GeV}$. For the generation of turbulence at first-order phase transitions, we have simply followed the same assumptions made in the literature regarding the dynamics of the phase transitions [57, 58, 60].

The turbulence generated can amplify tiny magnetic seed fields through the small-scale dynamo (SSD) mechanism. If we assume that the plasma is already weakly magnetized by the time of the EW scale, then SSD amplification will inevitable occur. The rapid amplification ends at saturation when the magnetic and kinetic energies are in approximate equipartition $E_{\mathrm{M}} / E_{\text {kin }} \approx \varepsilon$, where the efficiency of the mechanism is characterized by $\varepsilon$. The saturation efficiency parameter $\varepsilon$, which is determined numerically, varies depending on the type of forcing. For rotational modes $\varepsilon \approx 1$, whereas the saturation level is lower for compressive modes $\varepsilon \sim 10^{-3}-10^{-4}$ [35]. 
We note that these numerical studies were carried out for $P_{m} \approx 2$, further numerical work is required to establish the saturation level for large Prandtl numbers.

In this paper, we show that, even for tiny seed fields of strengths $B_{0}^{\text {seed }} \simeq\left(10^{-30}-10^{-20}\right) \mathrm{nG}$, the SSD mechanism can operate for a long enough period of time and be efficient enough to amplify such fields to saturation. The magnetic field strength saturates at $a^{2} B_{\mathrm{rms}} \sim 1 \varepsilon^{1 / 2} \mathrm{nG}$ on scales at most $\lambda_{c} \sim 10^{-5} \mathrm{pc}$ for turbulence generated by the primordial density perturbation. Such fields, assumed to be nonhelical, evolve to $B_{0}^{\mathrm{rms}} \sim 10^{-6} \varepsilon^{1 / 2} \mathrm{nG}$ on scales $\lambda_{c} \sim 10^{-1} \mathrm{pc}$ due to free turbulent decay. For turbulence generated by first-order phase transitions, the SSD mechanism can be even more effective, since the turbulent velocities can be quite large compared to those generated by the primordial density perturbation. We show that the mechanism can amplify magnetic fields to strengths $a^{2} B_{\mathrm{rms}} \sim\left(10^{-3}-1\right) \varepsilon^{1 / 2} \mu \mathrm{G}$ on scales $\lambda_{c} \sim\left(10^{-4}-10^{-1}\right) \mathrm{pc}$. These initial field configurations evolve to $a^{2} B_{\mathrm{rms}} \sim\left(10^{-6}-10^{-3}\right) \varepsilon^{1 / 2} \quad \mathrm{nG}$ on scales $\lambda_{c} \sim\left(10^{-1}-10^{2}\right)$ pc due to free turbulent decay.

Unfortunately, the damping of velocity perturbations due to neutrino diffusion inhibits turbulence from developing below the $\mathrm{QCD}$ scale $T_{\mathrm{QCD}} \simeq 200 \mathrm{MeV}$ (unless there is an injection of kinetic energy from a firstorder phase transition prior to neutrino decoupling at $T \gtrsim 2.6 \mathrm{MeV}$, see Fig. 2). Hence, it is difficult to generate turbulence from these mechanisms on larger length scales than $l_{c} \sim 10^{-1} \mathrm{pc}$. Without turbulence there is no SSD action and therefore no amplification of primordial magnetic fields on larger scales.

Although turbulent velocities are completely erased in viscous and free-streaming regimes, magnetic fields are overdamped and can survive to the present day. Such fields would fill the voids in the large scale structure and provide the seeds for magnetic fields generated by structure formation and galactic dynamo. Unfortunately, the saturated field strengths due to turbulence generated by the primordial density perturbation are too weak on too short scales in the voids of the large scale structure to explain the Fermi observations of TeV Blazars [6]. However, the field strengths obtained due to turbulence generated by first-order phase transitions are strong enough to explain such observations.

\section{DISCUSSION AND CONCLUSION}

The discrepancy between theoretically generated and observed magnetic fields in the Universe needs explaining. The galactic dynamo can be a very effective mechanism at producing the $\mu \mathrm{G}$ fields observed in spiral galaxies [1. However, strong fields in young galaxies, clusters and superclusters of galaxies and in the intergalactic medium require further explanation [2 6]. As noted in a number of numerical and analytical works, the rapid amplification of magnetic seed fields can occur due to the turbulent motions of the conducting plasma. This small-scale dynamo (SSD) mechanism is believed to play a crucial role in the formation of large magnetic fields in a number of astronomical settings, from stars to galaxies and the intergalactic medium [19,21, 39, 40]. For these settings, the turbulent motions arise from gravitational collapse, accretion and supernovae explosions. Hence, the SSD mechanism can be highly effective at magnetizing structures in the early Universe. However, the large field strengths apparently observed in the voids of the large-scale structure [6] still require an explanation.

Magnetic seed fields will almost certainly be generated at some level in the early Universe through a variety of mechanisms. Such mechanisms include inflation 9, phase transitions [10] and the Harrison mechanism through the generation of vorticity [16]. The SSD mechanism for the amplification of such seed fields could play an important role for the explanation of the observed large magnetic fields throughout the Universe. In this paper we have demonstrated that the conditions necessary for such turbulent amplification arise in the radiation dominated Universe before the onset of structure formation. We have shown that significant turbulence is generated in this early epoch by at least two mechanisms; velocity perturbations generated by the primordial density perturbation and bubble collisions in firstorder phase transitions.

Turbulent plasma motions arise inevitably from perturbations of the gravitational potential. The continuous production of velocity perturbations upon horizon entry of primordial density modes, act as a continuous forcing of the fluid on the largest scales. Therefore, in regimes of large Reynolds numbers, a state of stationary fully developed turbulence is expected. Turbulent flow can be triggered, for example, by thermal fluctuations on very small scales 34. Turbulence can also be injected into the plasma by bubble collisions during first-order phase transitions. Although the kinetic energy injection occurs only for the duration of the phase transition, we argue, following Refs. 58, 60, that a state of fully developed turbulence is also expected from this mechanism.

Once fully developed turbulence is established, the Kazantsev model of the SSD mechanism can be used to estimate the magnetic field growth rate. We have demonstrated that the Prandtl numbers are very large in the regime considered. Thus, the results from the Kazantsev theory for $P_{m} \gg 1$ are applicable. The analytical work shows that the magnetic field growth rate depends on the kinetic Reynolds numbers [36, 46, which are very large in our case. We have shown that, for both models of turbulence, the amplification is strong enough for small magnetic seed fields to reach a saturated state. The saturated state is given by the approximate equipartition between magnetic and kinetic energy $E_{\mathrm{M}} / E_{\mathrm{kin}} \approx \varepsilon$, where the parameter $\varepsilon$ characterizes the efficiency of the mechanism.

We note that numerical studies at Prandtl numbers $P_{m} \approx 2$ indicate that the SSD mechanism is more efficient for rotational modes, where the saturation efficiency 
$\varepsilon$ is close to unity 35 . Whereas the saturation level is lower for compressive modes $\varepsilon \sim 10^{-3}-10^{-4} 35$. However, further numerical work is required to establish the saturation level for larger Prandtl numbers and smaller Mach numbers relevant to our settings. We also note that, although only longitudinal velocity modes are generated by first-order primordial density perturbations, rotational modes are generated at second order in cosmological perturbations 51 55]. Also, there is no reason not to expect rotational modes generated by firstorder phase transitions. In any case, since the Reynolds numbers are so large, nonlinear interactions can play a role leading to a state of fully developed turbulence with both rotational and longitudinal modes. In particular, we expect that, below the integral scale, Kolmogorov type turbulence is established. But we stress that the SSD mechanism works independently of the type of turbulence [36, 45, 46. Indeed, even purely irrotational turbulence can still drive a small-scale dynamo [35, 36, 46. . Hence, the efficient amplification of magnetic fields seems unavoidable, leading to a strongly magnetized early Universe prior to structure formation.

For the two mechanisms of turbulence investigated in this paper, we calculated the saturated field strengths and their subsequent evolution up to the present day. We note that although turbulence is completely erased in viscous and free-streaming regimes, magnetic fields are overdamped and can survive to the present day. Therefore, the most important epochs of evolution are due to free turbulent decay. This turbulent MHD effect decreases the field strength and increases the coherence length in nonhelical fields [30, 63. From the turbu- lence generated by the primordial density perturbation we found $B_{0}^{\mathrm{rms}} \sim 10^{-6} \varepsilon^{1 / 2} \mathrm{nG}$ on scales $\lambda_{c} \sim 10^{-1} \mathrm{pc}$. Unfortunately, even for a high efficiency factor $\varepsilon \sim 1$, these fields are too weak on too short scales to explain the Fermi observations of TeV Blazars [6]. From the turbulence generated by first-order phase transitions, we found $B_{0}^{\text {rms }} \sim\left(10^{-6}-10^{-3}\right) \varepsilon^{1 / 2} \mathrm{nG}$ on scales $\lambda_{c} \sim\left(10^{-1}-10^{2}\right) \mathrm{pc}$. Such fields are strong enough to explain the apparent observations of intergalactic magnetic fields suggested by the Fermi results [6]. Thus, in this paper we have demonstrated that the conditions are right for the efficient amplification of magnetic fields via the small-scale dynamo. The mechanism generates large field strengths, albeit on very small scales, which could explain observations of magnetic fields in the voids of the large-scale structure and have an impact on early structure formation.

\section{ACKNOWLEDGMENTS}

We wish to thank the anonymous referees for their constructive comments. This work was supported by the Deutsche Forschungsgemeinschaft through the collaborative research centre SFB 676, by the Helmholtz Alliance for Astroparticle Phyics (HAP) funded by the Initiative and Networking Fund of the Helmholtz Association. DRGS thanks for funding by the German Science Foundation (DFG) via the SFB 963/1 Astrophysical Flow Instabilities and Turbulence (project A12).
[1] R. Beck, Space Science Reviews 166, 215 (2012)

[2] M. L. Bernet, F. Miniati, S. J. Lilly, P. P. Kronberg, and M. Dessauges-Zavadsky, Nature 454, 302 (2008), arXiv:0807.3347

[3] L. Feretti, G. Giovannini, F. Govoni, and M. Murgia, A\&AR 20, 54 (2012), arXiv:1205.1919 [astro-ph.CO]

[4] Y. Xu, P. P. Kronberg, S. Habib, and Q. W. Dufton, AstrophysJ 637, 19 (2006), arXiv:astro-ph/0509826

[5] S. Chakraborti, N. Yadav, C. Cardamone, and A. Ray, Astrophys. J. 746, L6 (Feb. 2012), arXiv:1110.3312 [astro-ph.CO]

[6] A. Neronov and I. Vovk, Science 328, 73 (2010), arXiv:1006.3504 [astro-ph.HE]

[7] A. E. Broderick, P. Chang, and C. Pfrommer, Astrophys. J. 752, 22 (Jun. 2012), arXiv:1106.5494 [astro-ph.CO]

[8] R. Schlickeiser, D. Ibscher, and M. Supsar, Astrophys. J. 758, 102 (Oct. 2012)

[9] M. S. Turner and L. M. Widrow, Phys.Rev. D37, 2743 (1988)

[10] G. Sigl, A. V. Olinto, and K. Jedamzik, Phys.Rev. D55, 4582 (1997), arXiv:astro-ph/9610201 [astro-ph]

[11] A. D. Dolgov and D. Grasso, Phys.Rev.Lett. 88, 011301 (2001), arXiv:astro-ph/0106154 [astro-ph]

[12] S. Matarrese, S. Mollerach, A. Notari, and A. Riotto, Phys.Rev. D71, 043502 (2005), arXiv:astro-ph/0410687 [astro-ph]

[13] K. Takahashi, K. Ichiki, H. Ohno, and H. Hanayama, Phys.Rev.Lett. 95, 121301 (2005), arXiv:astroph/0502283 [astro-ph]

[14] T. Kobayashi, R. Maartens, T. Shiromizu, and K. Takahashi, Phys.Rev. D75, 103501 (2007), arXiv:astro$\mathrm{ph} / 0701596$ [astro-ph]

[15] E. Fenu, C. Pitrou, and R. Maartens, Mon.Not.Roy.Astron.Soc. 414, $2354 \quad$ (2011), arXiv:1012.2958 [astro-ph.CO]

[16] E. Harrison, Mon.Not.Roy.Astron.Soc. 147, 279 (1970)

[17] A. Brandenburg and K. Subramanian, Phys.Rept. 417, 1 (2005), arXiv:astro-ph/0405052 [astro-ph]

[18] A.-C. Davis, M. Lilley, and O. Tornkvist, Phys.Rev. D60, 021301 (1999), arXiv:astro-ph/9904022 [astro-ph]

[19] D. R. G. Schleicher, R. Banerjee, S. Sur, T. G. Arshakian, R. S. Klessen, R. Beck, and M. Spaans, A\&A 522, A115 (Nov. 2010), arXiv:1003.1135 [astro-ph.CO]

[20] J. Schober, D. R. G. Schleicher, and R. S. Klessen, A\&A 560, A87 (Dec. 2013), arXiv:1310.0853 [astro-ph.GA]

[21] R. Beck, A. Brandenburg, D. Moss, A. Shukurov, and D. Sokoloff, ARA\&A 34, 155 (1996)

[22] D. Balsara, R. A. Benjamin, and D. P. Cox, Astrophys.J. 563, 800 (Dec. 2001), astro-ph/0107345

[23] S. Sur, C. Federrath, D. R. G. Schleicher, R. Baner- 
jee, and R. S. Klessen, MNRAS 423, 3148 (Jul. 2012), arXiv:1202.3206 [astro-ph.SR]

[24] A. M. Beck, K. Dolag, H. Lesch, and P. P. Kronberg, MNRAS 435, 3575 (Nov. 2013), arXiv:1308.3440 [astroph.GA]

[25] D. Seifried, R. Banerjee, and D. Schleicher, MNRAS 440, 24 (Mar. 2014), arXiv:1311.4991 [astro-ph.GA]

[26] D. R. G. Schleicher and R. Beck, A\&A 556, A142 (Aug. 2013), arXiv:1306.6652 [astro-ph.CO]

[27] G. K. Batchelor, Royal Society of London Proceedings Series A 201, 405 (Apr. 1950)

[28] A. Kazantsev, JHEP 53, 1806 (1967)

[29] E. W. Kolb and M. S. Turner, The Early universe (Addison-Wesley, 1990)

[30] R. Banerjee and K. Jedamzik, Phys.Rev. D70, 123003 (2004), arXiv:astro-ph/0410032 [astro-ph]

[31] K. Jedamzik, V. Katalinic, and A. V. Olinto, Phys.Rev. D57, 3264 (1998), arXiv:astro-ph/9606080 [astro-ph]

[32] Landau and Lifschitz, Vol. 6. Fluid mechanics (Pergamon Press, 1987)

[33] U. Frisch, Turbulence, the legacy of A.N. Kolmogorov (Cambridge University Press, 1995)

[34] S. Tsugé, Physics of Fluids 17, 22 (Jan. 1974)

[35] C. Federrath, G. Chabrier, J. Schober, R. Banerjee, R. S. Klessen, et al., Phys.Rev.Lett. 107, 114504 (2011), arXiv:1109.1760 [physics.flu-dyn]

[36] J. Schober, D. Schleicher, C. Federrath, R. Klessen, and R. Banerjee, Phys.Rev. E 85, 026303 (Feb. 2012), arXiv:1109.4571 [astro-ph.CO]

[37] J. Schober, D. Schleicher, S. Bovino, and R. S. Klessen, Phys. Rev. E 86, 066412 (Dec. 2012), arXiv:1212.5979 [astro-ph.CO]

[38] C. Federrath, S. Sur, D. R. G. Schleicher, R. Banerjee, and R. S. Klessen, Astrophys.J. 731, 62 (Apr. 2011), arXiv:1102.0266 [astro-ph.SR]

[39] M. Latif, D. Schleicher, W. Schmidt, and J. Niemeyer(2012), arXiv:1212.1619 [astro-ph.CO]

[40] S. Sur, D. R. G. Schleicher, R. Banerjee, C. Federrath, and R. S. Klessen, Astrophys. J., Letters 721, L134 (2010), arXiv:1008.3481 [astro-ph.CO]

[41] L. Haugen, A. Brandenburg, and W. Dobler, Astrophys.Space Sci. 292, 53 (2004), arXiv:astro-ph/0306453 astro-ph]

[42] K. Subramanian(1997), arXiv:astro-ph/9708216 [astro$\mathrm{ph}]$

[43] S. Bovino, D. R. G. Schleicher, and J. Schober, New Journal of Physics 15, 013055 (Jan. 2013), arXiv:1212.3419 [astro-ph.CO]

[44] D. R. Schleicher, J. Schober, C. Federrath, S. Bovino, and W. Schmidt, New J.Phys. 15, 023017 (2013),
arXiv:1301.4371 [astro-ph.CO]

[45] A. Gruzinov, S. C. Cowley, and R. Sudan, Phys.Rev.Lett. 77, 4342 (1996), arXiv:astro-ph/9611194 [astro-ph]

[46] A. A. Schekochihin, S. A. Boldyrev, and R. M. Kulsrud, Astrophys.J. 567, 828 (Mar. 2002), arXiv:astro$\mathrm{ph} / 0103333$

[47] K. Jedamzik and G. M. Fuller, Astrophys.J. 423, 33 (1994), arXiv:astro-ph/9312063 [astro-ph]

[48] P. Ade et al. (Planck Collaboration)(2013), arXiv:1303.5076 [astro-ph.CO]

[49] D. H. Lyth and A. R. Liddle, The primordial density perturbation: Cosmology, inflation and the origin of structure (Cambridge University Press, 2009)

[50] H. Kodama and M. Sasaki, Prog.Theor.Phys.Suppl. 78, 1 (1984)

[51] A. Lewis, Phys.Rev. D70, 043518 (2004), arXiv:astroph/0403583 [astro-ph]

[52] T. H.-C. Lu, K. Ananda, and C. Clarkson, Phys.Rev. D77, 043523 (2008), arXiv:0709.1619 [astro-ph]

[53] T. H.-C. Lu, K. Ananda, C. Clarkson, and R. Maartens, JCAP 0902, 023 (2009), arXiv:0812.1349 [astro-ph]

[54] A. J. Christopherson and K. A. Malik, Class.Quant.Grav. 28, 114004 (2011), arXiv:1010.4885 [gr-qc]

[55] K. Ichiki, K. Takahashi, and N. Sugiyama, Phys.Rev. D85, 043009 (2012), arXiv:1112.4705 [astro-ph.CO]

[56] P. J. Steinhardt, Phys.Rev. D25, 2074 (1982)

[57] M. Kamionkowski, A. Kosowsky, and M. S. Turner, Phys.Rev. D49, 2837 (1994), arXiv:astro-ph/9310044 [astro-ph]

[58] A. Kosowsky, A. Mack, and T. Kahniashvili, Phys.Rev. D66, 024030 (2002), arXiv:astro-ph/0111483 [astro-ph]

[59] M. Kamionkowski and K. Freese, Phys.Rev.Lett. 69, 2743 (1992), arXiv:hep-ph/9208202 [hep-ph]

[60] C. Caprini and R. Durrer, Phys.Rev. D74, 063521 (2006), arXiv:astro-ph/0603476 [astro-ph]

[61] M. Hindmarsh, S. J. Huber, K. Rummukainen, and D. J. Weir(2013), arXiv:1304.2433 [hep-ph]

[62] S. Dodelson, Modern cosmology (Academic Press, 2003)

[63] R. Durrer and A. Neronov, Astron.Astrophys.Rev. 21, 62 (2013), arXiv:1303.7121 [astro-ph.CO]

[64] J. Ahonen and K. Enqvist, Phys.Lett. B382, 40 (1996), arXiv:hep-ph/9602357 [hep-ph]

[65] K. Jedamzik and G. Sigl, Phys.Rev. D83, 103005 (2011), arXiv:1012.4794 [astro-ph.CO]

[66] L. Campanelli, Phys.Rev.Lett. 98, 251302 (2007), arXiv:0705.2308 [astro-ph]

[67] A. Brandenburg, K. Enqvist, and P. Olesen, Phys.Rev. D54, 1291 (1996), arXiv:astro-ph/9602031 [astro-ph]

[68] R. Durrer and C. Caprini, JCAP 0311, 010 (2003), arXiv:astro-ph/0305059 [astro-ph] 